\title{
The effect of interference on temporal order memory for random and fixed sequences in nondemented older adults
}

\author{
Jerlyn C. Tolentino, ${ }^{1}$ Eva Pirogovsky, ${ }^{2}$ Trinh Luu, ${ }^{1}$ Chelsea K. Toner, ${ }^{1}$ and \\ Paul E. Gilbert ${ }^{1,2,3}$ \\ ${ }^{1}$ Department of Psychology, San Diego State University, San Diego, California 92182, USA; ${ }^{2}$ San Diego State University-University \\ of California San Diego Joint Doctoral Program in Clinical Psychology, San Diego, California 92120, USA
}

\begin{abstract}
Two experiments tested the effect of temporal interference on order memory for fixed and random sequences in young adults and nondemented older adults. The results demonstrate that temporal order memory for fixed and random sequences is impaired in nondemented older adults, particularly when temporal interference is high. However, temporal order memory for fixed sequences is comparable between older adults and young adults when temporal interference is minimized. The results suggest that temporal order memory is less efficient and more susceptible to interference in older adults, possibly due to impaired temporal pattern separation.
\end{abstract}

Impaired memory for the temporal order of events or items in sequence may have adverse consequences on daily living skills and may affect a variety of cognitive functions (Pirogovsky et al. 2009). A primary function of the prefrontal cortex may be to integrate temporal information for the attainment of prospective behavioral goals (Fuster 2001). The prefrontal cortex is suggested to temporally organize fragments of information that are distributed spatially across cortical networks (Fuster 2001) and may support memory for the temporal order of items in a sequence (Shimamura 1995). Evidence from studies involving humans with frontal lobe damage (Milner et al. 1985; Daum and Mayes 2000) and studies using functional neuroimaging (Cabeza et al. 2000; Hayes et al. 2004; Knutson et al. 2004) have demonstrated that the frontal lobes play a critical role in memory for temporal sequences. In addition, several animal studies have reported that the frontal lobes are important for temporal order memory in nonhuman primates (Inoue and Mikami 2006) and rats (Hannesson et al. 2004). Studies involving humans with damage to the temporal lobes (Hopkins et al. 1995; Mayes et al. 2001), functional neuroimaging studies in humans (Ekstrom and Bookheimer 2007; Lehn et al. 2009), and studies in rats (Gilbert et al. 2001; Fortin et al. 2002; Manns et al. 2007; Howland et al. 2008) suggest that the temporal lobes also play a role in memory for the temporal order of items in a sequence. Therefore, as demonstrated recently by Devito and Eichenbaum (2011) and Ekstrom et al. (2011), there is evidence that both the frontal and temporal lobes are essential for temporal order memory.

Age-related changes in gray matter and white matter have been documented in many regions of the brain (Peters and Rosene 2003; Allen et al. 2005; Ziegler et al. 2010; Driscoll et al. 2009; Kennedy and Raz 2009), including those critical for temporal order memory such as medial temporal and frontal lobes. Specifically, volumetric reductions have been reported in the medial temporal and prefrontal regions of the human brain (Kramer et al. 2007; Fjell and Walhovd 2010; Raz et al. 2010; Cardenas et al. 2011). Age-related white matter changes also have been reported

\footnotetext{
${ }^{3}$ Corresponding author.
}

E-mail pgilbert@sciences.sdsu.edu.

Article is online at http://www.learnmem.org/cgi/doi/10.1101//m.026062.112. in frontal and temporal regions of the human brain (Davis et al. 2009; Salat et al. 2009; Murray et al. 2010; Ziegler et al. 2010). These structural changes in gray and white matter have been associated with age-related cognitive decline (Kramer et al. 2007; Fjell and Walhovd 2010; Murray et al. 2010; Ziegler et al. 2010; Cardenas et al. 2011).

Given the structural changes in the frontal and temporal regions of the brain, it is not surprising that studies have reported significant age-related impairments in the ability to encode, organize, and accurately retrieve the sequential order of stimuli and events (Parkin et al. 1995; Fabiani and Friedman 1997; Trott et al. 1999; Newman et al. 2001; Kessels et al. 2007; Old and Naveh-Benjamin 2008; Ulbrich et al. 2009). However, normal temporal order memory also has been reported in older adults (Sekuler et al. 2006). It is possible that the level of temporal interference generated in these various tasks may influence the performance of older adults. For example, studies have shown that items occurring further apart in a temporal sequence are easier to remember than items that are temporally adjacent in both humans and animals (for review, see Kesner and Hopkins 2006). This phenomenon is assumed to occur because there is more interference and a greater need to separate temporally proximal stimuli than temporally distant stimuli in a sequence (Gilbert et al. 2001). Although studies have examined temporal order memory in healthy older adults, few if any studies to date have conducted systematic manipulations of temporal interference.

Pattern separation is a mechanism for separating partially overlapping patterns of activation so that one pattern may be retrieved as separate from other patterns. A pattern separation mechanism may reduce interference among similar memory representations and increase the likelihood of accurate encoding and retrieval (Gilbert and Brushfield 2009). The hippocampus, and specifically the dentate gyrus (DG) and CA3 subregions, have been reported to support pattern separation (for review, see Kesner 2007; Gilbert and Brushfield 2009; Rolls 2010; Yassa and Stark 2011; Schmidt et al. 2012). Age-related changes in the DG and CA3 hippocampal subregions have been hypothesized to result in less-efficient pattern separation due to strengthened processing of stored information at the expense of processing new information (Wilson et al. 2006; Yassa et al. 2011). In support of 
this hypothesis, studies have reported impaired pattern separation for visual and spatial information in older adults (Toner et al. 2009; Stark et al. 2010; Yassa et al. 2010; Holden et al. 2012). However, no studies have examined age-related changes in pattern separation for temporal sequences of stimuli in humans. The present study investigated the effects of varying levels of interference on temporal order memory for random and fixed sequences of visuospatial stimuli in young and older adults. As described below, interference was manipulated by systematically varying the temporal separation between items in a sequence.

The sample consisted of 40 nondemented older adults over the age of 65 and 40 young adults between the ages of 18 and 25. Demographic data are provided in Table 1. Older adults were community-dwelling individuals who were screened for dementia using the Dementia Rating Scale (DRS) (Mattis 1976). The average DRS score for the older adults was $135.66(S E=0.81)$. A task published by Pirogovsky et al. (2009) was used to assess the effects of interference on temporal order memory for random sequences of visuospatial stimuli. The participant was seated $\sim 60 \mathrm{~cm}$ from a computer monitor. At the beginning of each trial, the participant was prompted to focus on the monitor where a computerized version of a radial 8-arm maze was presented. The participant was told that a circle would appear at the end of each arm one at a time and he/she should remember the sequence in which the circles were presented.

Each trial consisted of a sample phase followed by a choice phase. On the sample phase, a gray circle (3-cm diameter) appeared at the end of a randomly selected arm for $2 \mathrm{sec}$, and then the display was masked for 2 sec by a gray mask to eliminate afterimage effects. Then, another circle appeared at the end of a different randomly selected arm for $2 \mathrm{sec}$, followed by a 2 -sec mask. This continued until a circle had been presented once at the end of each of the eight arms in a random sequence that varied on each trial (Fig. 1A). On the choice phase, the participant was presented simultaneously with two circles for $5 \mathrm{sec}$, one at the end of one study phase arm and the other at the end of another study phase arm. The participant was asked to indicate which circle appeared earlier in the sequence.

Temporal separations of $0,2,4$, and 6 lags were randomly selected for each choice phase and represented the number of circles that occurred during the sample-phase sequence between the two circles presented simultaneously during the choice phase (Fig. 1B). For example, a 6-lag separation trial would consist of two choice-phase circles that occurred with six circles between them during the sample-phase sequence (e.g., first circle vs. eighth cir-

Table 1. Demographic data for the young adults and older adults tested on the random and fixed sequence temporal order memory tasks

\begin{tabular}{|c|c|c|c|c|}
\hline & \multicolumn{2}{|c|}{ Random sequence task } & \multicolumn{2}{|c|}{ Fixed sequence task } \\
\hline & Young adults & Older adults & Young adults & Older adults \\
\hline Age (years) & $18.55(0.21)$ & $75.65(1.62)$ & $19.40(0.36)$ & $74.15(1.67)$ \\
\hline $\begin{array}{l}\text { Gender } \\
(\mathrm{M} / \mathrm{F})\end{array}$ & $6 / 14^{\mathrm{a}}$ & $10 / 10^{\mathrm{a}}$ & $11 / 9^{\mathrm{b}}$ & $8 / 12^{\mathrm{b}}$ \\
\hline $\begin{array}{l}\text { Education } \\
\text { (years) }\end{array}$ & $13.40(0.17)^{\mathrm{c}}$ & $15.8(0.68)^{c}$ & $14.35(0.35)^{\mathrm{d}}$ & $16.55(0.59)^{d}$ \\
\hline
\end{tabular}

${ }^{\mathrm{a} A} \chi^{2}$ analysis did not reveal a significant gender difference between groups, $\chi^{2}(1, N=40)=1.67, P=0.20$.

${ }^{\mathrm{b}} \mathrm{A} \chi^{2}$ analysis did not reveal a significant gender difference between groups, $\chi^{2}(1, N=40)=0.90, P=0.34$.

${ }^{\mathrm{c}} \mathrm{A}$ one-way analysis of variance revealed that the older adults achieved more years of education than the young adults, $F_{(1,38)}=11.64, P<0.01$.

${ }^{\mathrm{d}} \mathrm{A}$ one-way analysis of variance revealed that the older adults achieved more years of education than the young adults, $F_{(1,38)}=6.93, P<0.05$.

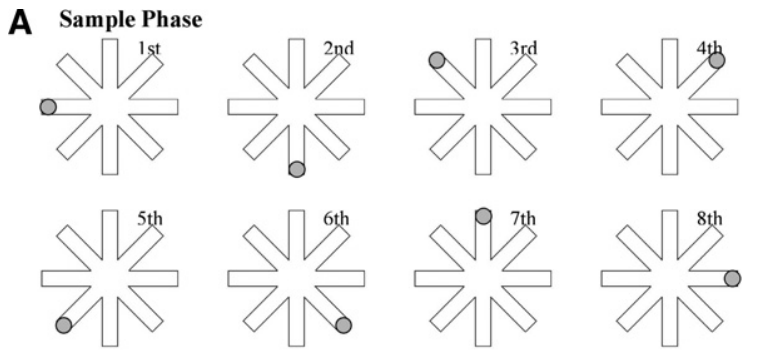

B Choice Phase

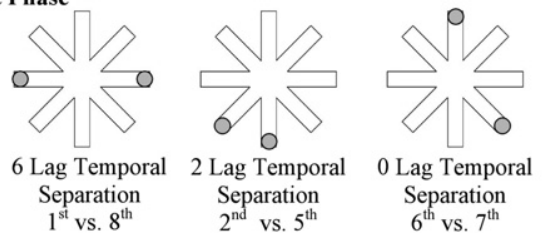

Figure 1. A schematic of a sample-phase temporal sequence showing locations of the first through the eighth arms presented in a sequence $(A)$ and a choice phase $(B)$ consisting of a 6-temporal separation lag trial, a 2temporal separation lag trial, and a 0-temporal separation lag trial.

cle presented). As reviewed above, evidence suggests that items occurring further apart in a temporal sequence are easier to remember than items that are temporally adjacent. Therefore, it was hypothesized that there was more interference and a greater need to separate temporally proximal circles on 0 and 2 lag trials than temporally distant circles on 4 and 6 lag trials. Following each sample-phase sequence, three choice phases were conducted involving three of the four temporal separations that were counterbalanced across sequences. Sixteen different sample-phase sequences were presented with three choice phases for each sequence. There were a total of 12 choice phase trials for each of the four temporal separations. A 15-sec intertrial interval was implemented between each trial.

An identical procedure was used to assess the effects of interference on temporal order memory for fixed sequences of visuospatial stimuli. However, on the fixed-sequence version of the task, the same sequence of eight gray circles paired with the same eight arms on the maze was presented on each trial.

Figure 2 (top) shows the mean $( \pm \mathrm{SE}$ ) percent correct performance of young and older adults on the random sequence temporal order memory task as a function of $0,2,4$, and 6 temporal separation lag trials. A $2 \times 4$ analysis of variance (ANOVA) with group (young, old) as the between-group factor and temporal separation lag $(0,2,4,6)$ as the within-group factor revealed significant main effects of group $F_{(1,38)}=27.57 ; P<0.001$ and temporal separation lag $F_{(3,114)}=19.42 ; P<0.001$. However, the group $\times$ temporal separation lag interaction was not significant $F_{(3,114)}=0.37 ; P=0.77$. The main effect of the group revealed that young adults significantly outperformed older adults $(P<$ 0.001). A Newman-Keuls post hoc comparison test of the temporal separation lag main effect revealed that 0 and 2 lag trials differed significantly $(P<0.05)$ from 4 and 6 lag trials.

Figure 2 (bottom) shows the mean $( \pm \mathrm{SE}$ ) percent correct performance of young and older adults on the fixed sequence temporal order task as a function of temporal separation lag. A $2 \times 4$ ANOVA revealed a significant main effects of group $F_{(1,38)}=$ 4.49, $P<0.05$ and temporal separation lag $F_{(3,114)}=13.60, P<$ 0.001 . In addition, the analysis revealed a significant group $\times$ temporal separation lag interaction $F_{(3,114)}=2.80, P<0.05$. A Newman-Keuls post hoc comparison test of the interaction demonstrated that young adults significantly outperformed older 
TEMPORAL ORDER MEMORY: RANDOM SEQUENCE

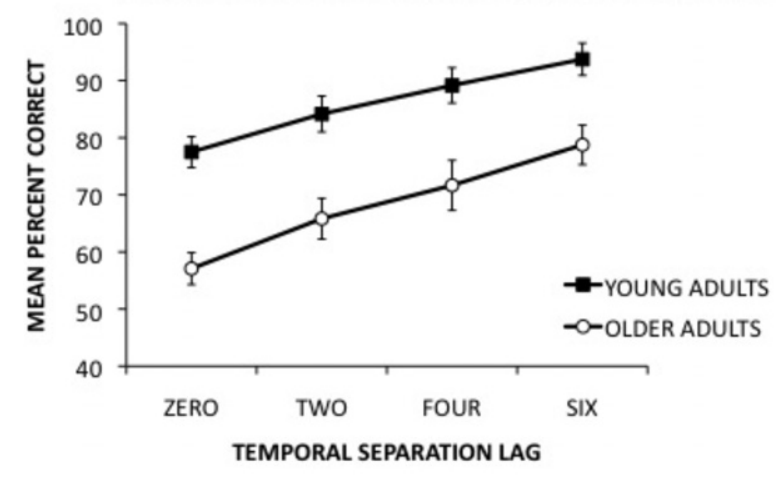

TEMPORAL ORDER MEMORY: FIXED SEQUENCE

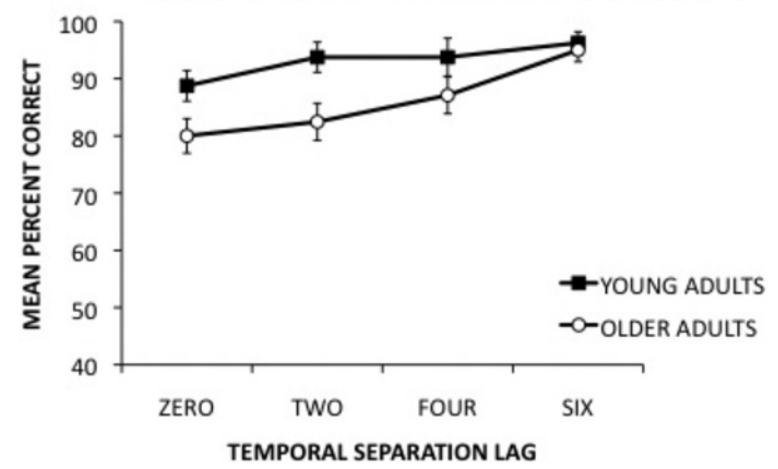

Figure 2. Mean $( \pm \mathrm{SE})$ percent correct performance of young and older adults on the random (top) and fixed (bottom) sequence temporal order memory task as a function of temporal separation lag $(0,2,4,6)$.

adults on temporal separation 0,2 , and 4 lag trials $(P<0.05)$. However, there were no significant differences between young and older adults on 6 lag temporal separation trials. A follow-up analysis examined task performance as a function of temporal separation lag across trials. The 48 trials were separated into two blocks of 24 trials. A $2 \times 2 \times 4$ ANOVA with group as a between group factor and block and lag as within group factors revealed significant main effects of group $F_{(3,38)}=4.18, P<0.05$, block $F_{(1,38)}=25.49, P<0.001$, and lag $F_{(3,114)}=12.76, P<0.001$, along with a group $\times$ lag interaction $F_{(3,114)}=3.35, P<0.05$. However, no significant interactions were found between block and group $(P=0.51)$ or lag $(P=0.36)$. These finding suggest that group differences across lags did not change significantly across trials. In addition, the performance of young adults on 6-lag trials was 96\% (shown in Fig. 2); therefore, it is possible that their performance had reached ceiling. However, this follow-up analysis revealed that age-related performance across lags on the first block of 24 trials (data not shown) was remarkably similar to the data shown in Figure 2. Importantly, the young group averaged only $92.5 \%$ on 6-lag trials on the first block of trials (well below ceiling), providing evidence that the similar performance level of young and older adults on 6-lag trials (Fig. 2) was unlikely due solely to ceiling effects.

For the present tasks, it is hypothesized that as temporal separation lag decreased (i.e., choice phase circles were closer together in time during the sample-phase sequence), interference was likely to increase, resulting in poorer temporal order memory. The results from the random sequence task demonstrate that the performance of young and older adults improved as a function of increased temporal separation lag and decreased temporal in- terference. However, young adults outperformed older adults across temporal separations with high, moderate, and low temporal interference. These findings suggest that temporal order memory for random sequences is less efficient in older adults compared with young adults.

The results from the fixed sequence task demonstrate that the performance of young and older adults also improved as a function of decreased temporal separation, presumably due to lessened temporal interference. Young adults outperformed older adults on trials involving high (e.g., 0 and 2 lags) and moderate temporal interference (e.g., 4 lag). However, there were no significant agerelated differences on trials with low interference (e.g., 6 lag). The data suggest that temporal order memory for fixed sequences is less efficient in older adults when temporal interference is high or moderate. However, their performance was comparable to young adults when interference was minimized. Given that participants viewed the fixed sequence 16 times, whereas each of the 16 random sequences was viewed once, the amount of training per sequence varied between the two tasks. Although future studies are needed to better examine the relationship between temporal interference and the amount of training on a sequence, an analysis of the present data (data not shown due to space limitations) did not reveal a significant temporal lag $\times$ task interaction, offering some preliminary indication that temporal interference did not interact with training on the sequences.

Disruption in sequence memory may affect various cognitive domains critical to the execution of daily living skills. Given that memory for temporally sequenced events, stimuli, or actions may be important for aspects of executive function (e.g., strategic planning, problem solving), temporal order memory impairment may contribute to executive dysfunction in older adults. In addition, temporal order memory for sequences also may be critical for episodic memory. One key feature of episodic memory is that elements must be associated into a context to demarcate the episode in space and time. The hippocampus may support mnemonic processes, such as temporal pattern separation, to enhance episodic memory accuracy. However, age-related changes in this region may have adverse effects on pattern separation.

Therefore, one possible interpretation of the present findings may suggest that pattern separation for temporal sequences is less efficient in older adults. Trials involving shorter temporal separation lags and greater temporal interference may have required the operation of a pattern separation mechanism to separate memory representations for the items in the sequence. This hypothesis is supported by the present data showing that performance on both tasks improved as a function of increased temporal separation lag. Since older adults were impaired across all temporal separation lags on both the tasks, with the exception of the 6 lag on the fixed task, it is possible that temporal pattern separation may be less efficient in older adults. However, when temporal interference was reduced on the 6-lag separation trials on the fixed sequence task, the need for pattern separation may have been minimized, resulting in optimized performance in older adults. Therefore, less-efficient pattern separation, potentially resulting from age-related changes in the hippocampus, may adversely affect temporal order memory for sequences of stimuli. Although studies reviewed above have provided evidence that pattern separation for visual object and spatial stimuli may be impaired in older humans, this may be the first study to demonstrate age-related changes in pattern separation for temporal sequences of stimuli. Behavioral interventions that minimize temporal interference and structure daily living tasks into repetitive, fixed sequences may improve memory and perhaps could increase functional independence in older adults. In addition, a recent study reports that impaired temporal order memory may be a selective behavioral marker of Alzheimer's disease (Bellassen et al. 2012). Therefore, 
the present findings potentially may have both basic science and clinical implications.

\section{Acknowledgments}

This research was supported by a National Institutes of Health grant (\#AG034202) to PEG from the National Institute on Aging. We thank all of the participants for their contributions to this study.

\section{References}

Allen JS, Bruss J, Brown CK, Damasio H. 2005. Normal neuroanatomical variation due to age: The major lobes and a parcellation of the temporal region. Neurobiol Aging 26: 1245-1260.

Bellassen V, Iglói K, de Souza LC, Dubois B, Rondi-Reig L. 2012. Temporal order memory assessed during spatiotemporal navigation as a behavioral cognitive marker for differential alzheimer's disease diagnosis. J Neurosci 32: 1942-1952.

Cabeza R, Anderson ND, Houle S, Mangels JA, Nyberg L. 2000. Age-related differences in neural activity during item and temporal-order memory retrieval: A positron emission tomography study. J Cogn Neurosci 12: 197-206.

Cardenas VA, Chao LL, Studholme C, Yaffe K, Miller BL, Madison C, Buckley ST, Mungas D, Schuff N, Weiner MW. 2011. Brain atrophy associated with baseline and longitudinal measures of cognition. Neurobiol Aging 32: 572-580.

Daum I, Mayes AR. 2000. Memory and executive function impairments after frontal or posterior cortex lesions. Behav Neurol 12: 161-173.

Davis SW, Dennis NA, Buchler NG, White LE, Madden DJ, Cabeza R. 2009. Assessing the effects of age on long white matter tracts using diffusion tensor tractography. Neuroimage 46: 530-541.

Devito LM, Eichenbaum H. 2011. Memory for the order of events in specific sequences: Contributions of the hippocampus and medial prefrontal cortex. J Neurosci 31: 3169-3175.

Driscoll I, Davatzikos C, An Y, Wu X, Shen D, Kraut M, Resnick SM. 2009. Longitudinal pattern of regional brain volume change differentiates normal aging from MCI. Neurology 72: 1906-1913.

Ekstrom AD, Bookheimer SY. 2007. Spatial and temporal episodic memory retrieval recruit dissociable functional networks in the human brain. Learn Mem 14: 645-654.

Ekstrom AD, Copara MS, Isham EA, Wang WC, Yonelinas AP. 2011. Dissociable networks involved in spatial and temporal order source retrieval. Neuroimage 56: 1803-1813.

Fabiani M, Friedman D. 1997. Dissociations between memory for temporal order and recognition memory in aging. Neuropsychologia 35: $129-141$.

Fjell AM, Walhovd KB. 2010. Structural brain changes in aging: Courses, causes and cognitive consequences. Rev Neurosci 21: 187-221.

Fortin NJ, Agster KL, Eichenbaum H. 2002. Critical role of the hippocampus in memory for sequences of events. Nat Neurosci 5: 458-462.

Fuster JM. 2001. The prefrontal cortex - An update: Time is of the essence. Neuron 30: 319-333.

Gilbert PE, Brushfield AM. 2009. The role of the CA3 hippocampal subregion in spatial memory: A process oriented behavioral assessment. Prog Neuropsychopharmacol Biol Psychiatry 33: 774-781.

Gilbert PE, Kesner RP, Lee I. 2001. Dissociating hippocampal subregions: A double dissociation between dentate gyrus and CA1. Hippocampus 11: 626-636.

Hannesson DK, Vacca G, Howland JG, Phillips AG. 2004. Medial prefrontal cortex is involved in spatial temporal order memory but not spatial recognition memory in tests relying on spontaneous exploration in rats. Behav Brain Res 153: $273-285$.

Hayes SM, Ryan L, Schnyer DM, Nadel L. 2004. An fMRI study of episodic memory: Retrieval of object, spatial, and temporal information. Behav Neurosci 18: 885-896.

Holden HM, Hoebel C, Loftis K, Gilbert PE. 2012. Spatial pattern separation in cognitively normal young and older adults. Hippocampus doi: 10.1002/hipo.22017.

Hopkins RO, Kesner RP, Goldstein M. 1995. Memory for novel and familiar spatial and linguistic temporal distance information in hypoxic subjects. J Int Neuropsychol Soc 1: 454-468.

Howland JG, Harrison RA, Hannesson DK, Phillips AG. 2008. Ventral hippocampal involvement in temporal order, but not recognition, memory for spatial information. Hippocampus 18: 251-257.

Inoue M, Mikami A. 2006. Prefrontal activity during serial probe reproduction task, encoding, mnemonic, and retrieval processes. J Neurophysiol 95: 1008-1041.
Kennedy KM, Raz N. 2009. Pattern of normal age-related regional differences in white matter microstructure is modified by vascular risk. Brain Res 1297: 41-56.

Kesner RP. 2007. Behavioral functions of the CA3 subregion of the hippocampus. Learn Mem 14: 771-781.

Kesner RP, Hopkins RO. 2006. Mnemonic functions of the hippocampus: A comparison between animals and humans. Biol Psych 73: 3-18.

Kessels RP, Hobbel D, Postma A. 2007. Aging, context memory and binding: A comparison of "what, where and when" in young and older adults. Int J Neurosci 117: 795-810.

Knutson KM, Wood JN, Grafman J. 2004. Brain activation in processing temporal sequence: An fMRI study. Neuroimage 23: 1299-1307.

Kramer JH, Mungas D, Reed BR, Wetzel ME, Burnett MM, Miller BL, Weiner MW, Chui HC. 2007. Longitudinal MRI and cognitive change in healthy elderly. Neuropsychology 21: 412-418.

Lehn H, Steffenach HA, van Strien NM, Veltman DJ, Witter MP, Haberg AK. 2009. A specific role of the human hippocampus in recall of temporal sequences. J Neurosci 29: 3475-3484.

Manns JR, Howard MW, Eichenbaum H. 2007. Gradual changes in hippocampal activity support remembering the order of events. Neuron 56: $530-540$

Mattis S. 1976. Mental status examination for organic mental syndrome in the elderly patient. In Geriatric psychiatry: A handbook for psychiatrists and primary care physicians (ed. Bellack L, Katsau TB), pp. 77-121. Grune \& Stratton, New York.

Mayes AR, Isaac CL, Holdstock JS, Hunkin NM, Montaldi D, Downes JJ, Macdonald C, Cezayirli E, Roberts JN. 2001. Memory for single items, word pairs, and temporal order in a patient with selective hippocampal lesions. Cogn Neuropsychol 18: 97-123.

Milner B, Petrides M, Smith ML. 1985. Frontal lobes and the temporal organization of memory. Human Neurobiol 4: 137-142.

Murray ME, Senjem ML, Peterson RC, Hollman JH, Preboske GM, Weigand SD, Knopman DS, Ferman TJ, Dickson DW, Jack CR Jr. 2010. Functional impact of white matter hyperintensities in cognitively normal elderly subjects. Arch Neurol 67: $1379-1385$

Newman MC, Allen JB, Kaszniak AW. 2001. Tasks for assessing memory for temporal order versus memory for items in aging. Aging Neuropsych Cogn 8: $72-78$.

Old SR, Naveh-Benjamin M. 2008. Memory for people and their actions: Further evidence for an age-related associative deficit. Psych Aging 23: 467-472.

Parkin AJ, Hunkin NM, Walter BM. 1995. Relationships between normal aging, frontal lobe function, and memory for temporal and spatial information. Neuropsychology 9: 304-312.

Peters A, Rosene DL. 2003. In aging, is it gray or white? J Comp Neurol 462: 139-143.

Pirogovsky E, Goldstein J, Peavy G, Jacobson MW, Corey-Bloom J, Gilbert PE. 2009. Temporal order memory deficits prior to clinical diagnosis in Huntington's disease. J Int Neuropsychol Soc 15: $662-670$.

Raz N, Ghisletta P, Rodrigue KM, Kennedy KM, Lindenberger U. 2010. Trajectories of brain aging in middle-aged and older adults: Regional and individual differences. Neuroimage 51: 501-511.

Rolls ET. 2010. A computational theory of episodic memory formation in the hippocampus. Behav Brain Res 215: 180-196.

Salat DH, Greve DN, Pacheco JL, Quinn BT, Helmer KG, Buckner RL, Fischl B. 2009. Regional white matter volume differences in nondemented aging and Alzheimer's disease. Neuroimage 44: 1247-1258.

Schmidt B, Marrone DF, Markus EJ. 2012. Disambiguating the similar: The dentate gyrus and pattern separation. Behav Brain Res 226: 56-65.

Sekuler R, McLaughlin C, Kahana MJ, Wingfield A, Yotsumoto Y. 2006. Short-term visual recognition and temporal order memory are both well-preserved in aging. Psychol Aging 21: 632-637.

Shimamura AP. 1995. Memory and the prefrontal cortex. Ann N Y Acad Sci 769: $151-159$.

Stark SM, Yassa MA, Stark CE. 2010. Individual differences in spatial pattern separation performance associated with healthy aging in humans. Learn Mem 17: 284-288.

Toner CK, Pirogovsky E, Kirwan CB, Gilbert PE. 2009. Visual object pattern separation deficits in nondemented older adults. Learn Mem 16: $338-342$.

Trott CT, Friedman D, Ritter W, Fabiani M, Snodgrass JG. 1999. Episodic priming and memory for temporal source: Event-related potentials reveal age-related differences in prefrontal functioning. Psychol Aging 14: $390-413$

Ulbrich P, Churran J, Fink M, Wittman M. 2009. Perception of temporal order: The effects of age, sex, and cognitive factors. Neuropsychol Dev Cogn Section B Aging Neuropsychology Cogn 16: 183-202. 
Wilson IA, Gallagher M, Eichenbaum H, Tanila H. 2006. Neurocognitive aging: Prior memories hinder new hippocampal encoding. Trends Neurosci 29: 662-670.

Yassa MA, Stark CE. 2011. Pattern separation in the hippocampus. Trends Neurosci 34: 515-525.

Yassa MA, Lacy JW, Stark SM, Albert MS, Gallagher M, Stark CE. 2010. Pattern separation deficits associated with increased hippocampal CA3 and dentate gyrus activity in nondemented older adults. Hipросатpus doi: $10.1002 /$ hipo. 20808 .
Yassa MA, Mattfeld AT, Stark SM, Stark CE. 2011. Age-related memory deficits linked to circuit-specific disruptions in the hippocampus. Proc Nati Acad Sci 108: 8873-8878.

Ziegler DA, Piguet O, Salat DH, Prince K, Connally E, Corkin S. 2010. Cognition in healthy aging is related to regional white matter integrity, but not cortical thickness. Neurobiol Aging 31: 1912-1926.

Received February 23, 2012; accepted in revised form April 12, 2012. 


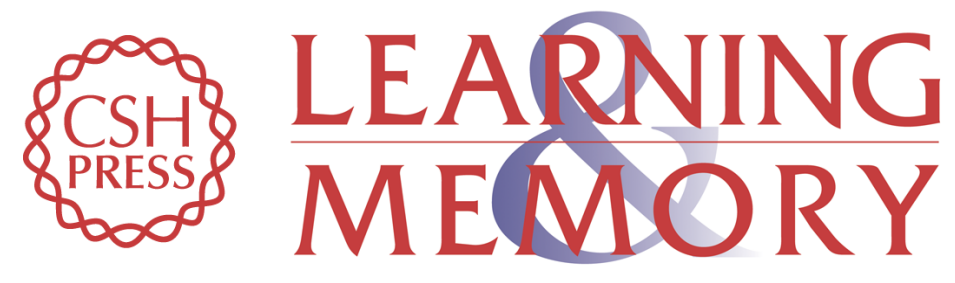

\section{The effect of interference on temporal order memory for random and fixed sequences in nondemented older adults}

Jerlyn C. Tolentino, Eva Pirogovsky, Trinh Luu, et al.

Learn. Mem. 2012, 19:

Access the most recent version at doi:10.1101/lm.026062.112

References This article cites 53 articles, 9 of which can be accessed free at:

http://learnmem.cshlp.org/content/19/6/251.full.html\#ref-list-1

License

Email Alerting Receive free email alerts when new articles cite this article - sign up in the box at the Service top right corner of the article or click here. 